\title{
Thin and superthin ion current sheets. Quasi-adiabatic and nona- diabatic models
}

\author{
L. M. Zelenyi' ${ }^{1}$, M. I. Sitnov ${ }^{2}$, H. V. Malova ${ }^{2}$, and A. S. Sharma ${ }^{3}$ \\ ${ }^{1}$ Space Research Institute, Moscow, Russia \\ ${ }^{2}$ Nuclear Physics Institute. Moscow State University, Moscow, Russia \\ *Present address: Department of Astronomy, University of Maryland. College Park, MD 20742, USA \\ ${ }^{3}$ Department of Astronomy, University of Maryland. College Park, MD 20742. USA
}

Received: 22 November 1999 - Revised: 23 March 2000 - Accepted: 6 April 2000

\begin{abstract}
Thin anisotropic current sheets (CSs) are phenomena of the general occurrence in the magnetospheric tail. We develop an analytical theory of the self-consistent thin CSs. General solitions of the GradShafranov equation are obtained in a quasi-adiabatic approximation which neglects the jumps of the sheet adiabatic invariant $I_{z}$. This is possible if the anisotropy of the initial distribution function is not too strong. The resulting structure of the thin CSs is interpreted as a sum of negative dia- and positive paramagnetic currents flowing near the neutral plane. In the immediate vicinity of the magnetic field reversal region the paramagnetic current arising from the meandering motion of the ions on Speiser orbits dominates. The maximum CS thickness is achieved in the case of weak plasma anisotropy and is of the order of the thermal ion gyroradius outside the sheet. A unified picture of thin CS scalings includes both the quasi-adiabatic regimes of weak and strong anisotropies and the nonadiabatic limit of superstrong anisotropy of the source ion distribution. The later limit corresponds to the case of almost field-aligned initial distribution, when the ratio of the drift velocity outside the CS to the thermal ion velocity exceeds the ratio of the magnetic field outside the CS to its value inside the $\operatorname{CS}\left(v_{D} / v_{T}>B_{0} / B_{n}\right)$. In this regime the jumps of $I_{z}$ become essential, and the current sheet thickness is approaching to some small but finite value, which depends upon the parameter $B_{n} / B_{0}$. Convective electric ficld increases the effective anisotropy of the source distribution and might produce the essential CS thinning which could have important implications for the substorm dynamics.
\end{abstract}

\section{Introduction}

Thin current sheets, i.e. the sheets with the thicknesses of the order of the ion gyroradius (or less) are frequently observed at different locations in the Earth's magnetotail, for example in the near-Earth and the midtail regions during the quiet times and the substorm growth phase (Fairfield, 1984; Kaufmann, 1987; McPherron et al., 1987; Mitchell et al., 1990; Sergeev et al., 1993; Sanny et al., 1994; Pulkkinen et al., 1998), and also in the distant region of the magnetotail (Pulkkinen et al., 1993). Mitchell et al. (1990) demonstrated that in the late growth phase the current sheet with $L \sim 400 \mathrm{~km}$ is dominated by cross-tail motion of quasi-adiabatic ions (Speiser, 1965). In the extensive study by Sergeev et al. (1998) no evidences were found of charged thin current sheet structures maintained by electrons. The important characteristic feature of thin current sheets is that their cross-tail current density profile doesn't coincide with and might be significantly more narrow than the profile of the plasma density (Sergeev et al., 1993; Sanny et al., 1994). A thin current sheets with the thicknesses of the order of $0.2 R_{E}$ or less could therefore be often embedded inside a few $R_{E}$ thick plasma sheet (PS).

These experimental investigations stimulated the development of the numerous mathematical models of thin current sheets in the magnetotail. Pritchett and Coroniti $(1994,1995)$ presented kinetic simulations of very thin (thickness less than a proton gyrodiameter) CSs with electrons as the dominant current carriers, which may exist in the magnetotail neutral sheet in the transition domain between dipole-like and tail-like regions. The results of this modeling were explained on the basis of the CS models with the nonzero electrostatic potential (Kuznetsova et al., 1998) and isotropic distribution function.

The analytical isotropic models where magnetic tension of curved field lines is balanced by the plasma pressure gradient (Schindler, 1974; Kan, 1973, Birn, 1987) 
were extensively exploited in magnetospheric physics for more than two decades. Another principal opportunity to balance the magnetic field line tension by centrifugal force exerted on finite mass particles moving along curved field lines was suggested in pioneer papers by Speiser (1965) and Eastwood (1972). Such model of CS balance implies the existence of the finite plasma anisotropy outside the sheet. This consideration was based on the existence of the so called Speiser orbits which were essentally nonadiabatic in the CS. The numerical kinetic modeling by Pritchett and Coroniti (1995) demonstrated that the ion CSs had well expressed diamagnetic "wings". Investigations of Holland and Chen (1993), Harold and Chen (1996) clearly demonstrated the important role of the magnetization currents in equilibrium CSs, particularly in the case of weak anisotropy. Recently Kaufmann (1997) tried to take into account the influence of non-guiding center particles on the structure of CS (particularly of trapped particles) and found that although the net currents carried by trapped particles is equal to zero, their existence could appreciably change the resulting CS structure. Previous non-selfconsistent studies of anisotropic ion CSs made by Alexeev and Malova (1990) demonstrated some of the characteristic features of the ion dominated anisotropic sheets, i.e. small $\left(\sim c / \omega_{p i}\right)$ scales and embedded structure. One further class of magnetotail models where equilibrium is maintained by the scattering of currentcarrying particles on the structured multiscale magnetic "turbulence" was proposed by Zelenyi et al. (1998).

The scaling of thin CSs is relatively well investigated. First, Francfort and Pellat (1976) made the non-selfconsistent estimate of the CS thickness $L$ in the limit of strong anisotropy: $L \approx \rho^{*}\left(v_{T} / v_{D}\right)^{4 / 3}$ where $v_{T}$ and $v_{D}$ are respectively the thermal ion velocity and the bulk speed of the ion flow outside the sheet and $\rho^{*}=$ $m v_{D} / e B_{0}$ is the Larmor radius and $v_{D} \gg v_{T}$. This estimate was confirmed later by Burkhart et al. (1992). The non-selfconsistent estimate of the weak anysotropic CS thickness was made by Ashour-Abdalla et al. (1994) who obtained $L \approx \rho_{0} \sim v_{T} / \omega_{0}$ ( $\rho_{0}$ is a thermal ion gyroradius for $v_{T} \geq v_{D}$ limit). Similar scaling have been also mentioned earlier by Chen et al. (1990). The particular case of the super-strong anisotropy (ion motion almost along the magnetic field lines) was considered by Pritchett and Coroniti (1992) and Burkhart et al. (1992). They found scaling of the CS in a form $L \approx \rho^{*} b_{n}^{4 / 3}$, where $b_{n}=B_{n} / B_{0}$ ( $B_{n}$ and $B_{0}$ are the magnetic fields at the plane of the sheet and and far outside of the shcet, respectively).

The first entirely self-consistent analytical thin CS model has been proposed by Kropotkin et al. (1997) for region of the moderately strong plasma anisotropy outside the thin CS $b_{n} \ll v_{T} / v_{D} \ll 1$. They assumed the conservation of the invariant

$I_{z}=\frac{1}{2 \pi} \oint m v_{z} d z$

(Speiser, 1970; Sonnerup, 1971; Büchner and Zelenyi, 1989) for this parameter range and confirmed the earlier non self-consistent estimates of the CS thickness (Francfort and Pellat, 1976). As a result they obtained the analytical solution for the magnetic field and current density profiles which could be considered as anisotropic analogue of the well-known Harris (1962) solution with $B_{n}=0$. In this paper our consideration is based on the more general model allowing the arbitrary ion population anisotropy outside CS (Sitnov et al., 2000).

We start from a brief description of the self-consistent CS theory based on the quasiadiabatic approximation for ion dynamics, which provides the conservation of the invariant (1). This assumption allows to obtain the equations of the Grad-Shafranov type for the ion dominated CSs. In the next part of the paper we explicitly take into account the nonadiabatic variation of the invariant $I_{z}$ and estimate the minimum thickness which could have self-consistent CS when the parameter $v_{D} / v_{T}$, characterizing the anisotropy, could be arbitrarily large. In the last section we tried to combine these two approaches and present the unified picture of thin quasi-adiabatic CS scales. Transforming our results to the corresponding deHoffmann-Teller coordinate frame we could easily generalize our consideration to the situation when constant dawn-dusk electric field is imposed on the system. In particular, it may affect strongly the thinning of the near-Earth tail current sheet with the interesting implications for substorm dynamics.

\section{Basic theory}

We start from the description of the basic self-consistent theory (Sitnov et al., 2000). The details may be found in Appendix 1. The CS is homogeneous along the EarthSun line $(X)$ and is infinite in the dawn-dusk $(Y)$ direction. The magnetic field has two components: $\mathrm{B}=(B(z)$, $\left.0, B_{n}\right)$ with $B_{0}=|B(z \rightarrow \pm \infty)|$ and electric field is set to zero by choosing deHoffmann-Teller frame of reference. In section 5 we will discuss how our results could be transformed to the magnetospheric frame. We also assume that the current is supported mainly by the ion population, and the dynamics of this population is quasi-adiabatic: $I_{z} \approx$ const (Büchner and Zelenyi, 1989). The latter assumption is valid under the condition

$\kappa=\sqrt{R_{c} / \rho_{z}} \ll 1$

where $\rho_{z}$ is the maximum gyroradius of the ion in the magnetic field at the location where magnetic field lines have the smallest curvature radius $R_{c}$. For the lypical magnetotail current sheet parameters: $B_{0} \sim 20 \mathrm{nT}$, 
$B_{n} \sim 1 n T$, thickness $L \sim 1000 \mathrm{~km}$ and ion energy about $3 \mathrm{keV}$ the value of ion $\kappa$ is of the order of 0.1 , i.e. the quasi-adiabaticity condition is justified.

The electron population is considered in the models as a charge neutralizing background making negligible contribution to the total current density. According to Shay et al. (1998), Hesse et al. (1996), and Nishikawa (1998), electrons may be essential current carriers in the small (of the order of the electron skin depth) vicinity of the $X$-line. Our model having everywhere the nonzero normal component of the magnetic field describes rather different CS configurations like the outflow region of the reconnection pattern. This is why the frozen-in condition holds for the electron species, and no considerable electron current is expected (see also Larsson and Kaufmann, 1996).

The quasi-adiabatic approximation of the ion dynamics allows us to use a new set of integrals of motion to avoid the solving of the Vlasov equation. We use in particular the quasi-adiabatic invariant $I_{z}$ defined by (1) and the total particle energy $W=m v_{0}^{2} / 2$. Conservation of quasi-adiabatic invariant (1) has been investigated in a number of papers (e.g., Büchner and Zelenyi, 1989), Zelenyi and Savenkov, 1993). In these studies the dimensionless value of adiabatic invariant $I_{z}$ was used: $I^{\prime}=(3 \pi / 8) I_{z} / v_{0} m \sqrt{L \rho_{0}}$, where $v_{0}$ is the total particle velocity, $m$ is the ion mass and $L$ is the sheet thickness. According to these papers, for sufficiently large values of $I^{\prime}>\kappa$ the medium value of the separatrix jumps of the invariant $I^{\prime}$ averaged over the ensemble of particles uniformly distributed over the initial phases (as it is implicitly assumed in our model) is precisely zero, while the mean square value of the jumps is equal to $\left\langle\left(\Delta I^{\prime}\right)^{2}\right\rangle=\left(3 \pi^{2} / 16\right) \cdot \kappa^{2} \cdot\left(1-\left(I^{\prime}\right)^{4 / 3}\right]$. So the average value of the jumps $\left\{\Delta I^{\prime} \mid\right.$ is of the order of $\kappa$ and the necessary condition when one could approximately neglect them $\left(I^{\prime} \gg\left|\Delta I^{\prime}\right|\right)$ is: $I^{\prime}>\kappa$. In our model this condition is equivalent to $\varepsilon>B_{n} / B_{0}$ where $\varepsilon=v_{T} / v_{D}$. As long as the latter condition holds, the invariant $I_{z}$ is approximately conserved. This simplification gives us the chance to consider the equilibrium problem analytically in a large interval of $\varepsilon$. The extended discussion of this issue is also given in section 4 of the paper by Kropotkin et al. (1997). Because our model has no explicit $y$-dependence: $\partial / \partial y \equiv 0$, the generalized momentum $P_{y}=v_{y}+(e / m c) A_{y}(x, z)$ provides the additional integral of motion.

As a result, the invariant (1) may be expressed in the form:

$$
I_{z}=\frac{2 m}{\pi} \int_{z_{0}}^{z_{1}} \sqrt{v_{y}^{2}+v_{z}^{2}-\left[v_{y}+\frac{e}{m c} \int_{z}^{z^{\prime}} B\left(z^{\prime \prime}\right) d z^{\prime \prime}\right]^{2}} d z^{\prime}
$$

where the limits of the integration $z_{0}$ and $z_{1}$ are given by the equation

$$
\frac{e}{m c} \int_{z}^{z_{0,1}} B\left(z^{\prime \prime}\right) d z^{\prime \prime}=\mp \sqrt{v_{y}^{2}+v_{z}^{2}}-v_{y}
$$

with the additional restriction $z_{0}=0$ if the formal solution $z_{0}$ of Eq.(4) turns out to be negative. The general structure of the distribution function could therefore be presented in a form

$f(z, \mathbf{v})=f\left(W\left(v_{x}, v_{y}, v_{z}\right), I_{z}\left(v_{x}, v_{y}, z, z_{0,1}\left(v_{x}, v_{y}, z\right)\right)\right)$

Basic dimensionless equations for the self-consistent magnetic field are given in the Appendix 1 (A3)-(A4). One can also investigate two different limits of these equations, i.e. the limits of strong and weak anisotropies (see the Appendix 2). It follows from (A11) that the scaling in the case of the strong anisotropy is $L \approx \rho^{*} \varepsilon^{4 / 3}$. Contrary to this case for the weak anisotropy the corresponding universal profiles of the current and magnetic field scale as $L \sim \varepsilon v_{D} / \omega_{0}=v_{T} / \omega_{0}=\rho_{0}$, so that the width of the weakly anisotropic sheet is always of order of the thermal ion gyroradius $\rho_{0}$. This result is consistent with the earlier estimate of the CS thickness (Ashour-Abdalla et al., 1994), made by considering the converging iterations to the possible self-consistent solution.

3 The role of dia- and paramagnetic currents in the formation of the embedded structure of CS

The general structure of the self-consistent magnetic field profile (A3) includes the contributions of partial dia- and paramagnetic currents along $Y$-axis. The inportant role of the magnetization currents was underlined by investigations of Holland and Chen (1993), Harold and Chen (1996) who have found numerically that plasma diamagnetism can play the significant role for the case $v_{D} \ll v_{T}$ but is quite small in the opposite case.

Schematics of the cross-tail currents is shown in Fig. 1. In general, one can split the total diamagnetic current outside the sheet, where the particle motion could be roughly considered as magnetized, into several "components" (Frank-Kamenezkii, 1968):

$\vec{\jmath}_{\perp}=\frac{c}{B_{0}^{2}}\left(\frac{p_{\perp}}{B_{0}}\left[\vec{B}_{0} \times \vec{\nabla} B_{0}\right]+\frac{p_{\|}}{R^{2}}\left[\vec{R} \times \vec{B}_{0}\right]\right)-\operatorname{crot} \frac{p_{\perp}}{B_{0}^{2}} \vec{B}_{0}$

where $p_{\perp}=n m v_{\perp}^{2} / 2, p_{\|}=n m v_{\|}^{2}, R-$ is the curvature radius of magnetic field lines. The resulting current is the combination of gradient (first term in the R.H.S. of Eq.(6)), centrifugal, or curvature (second term), and magnetization currents (third one). Their sum manifests the natural plasma diamagnetism and provides the total current in the negative $Y$-direction. Region A (having dark gray-shading in Fig. 1) corresponds to 


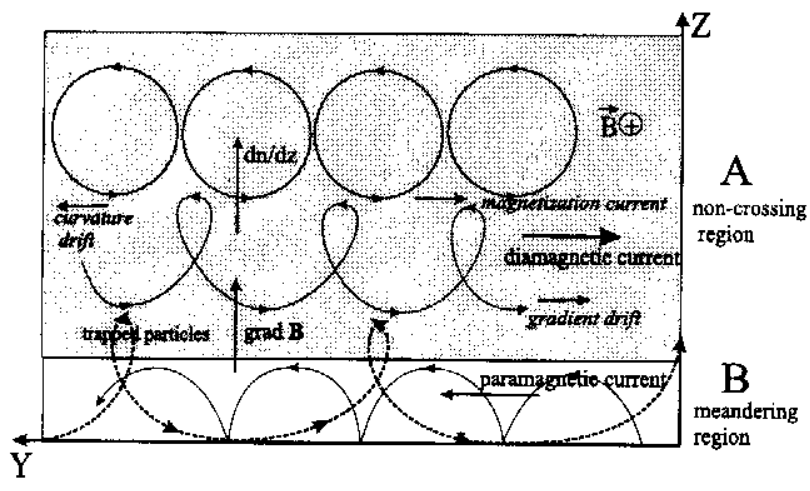

Fig. 1. Main constituents of the cross-tail current: A. Noncrossing region, where guiding center approximation (Eqs.(6)-(7)) could be applied. Schematically are shown: Larmor rotation of particles and their gradient (first term in Eq.(6)), curvature (second term) and magnetization (third term) drifts. Net current in this region is negative which illustrates the natural diamagnetism of magnetized plasma. B. Meandering region of non-guiding center motion. Positive paramagnetic (edgc) current is carried by meandering segments of the transit (Speiser) orbits. Particles trapped within CS on specific meandering orbits carry the negative current near the plane $z=0$ and positive current at the edges of their trapping region. At the edges of CS partial compensation of paramagnetic current by diamagnetic drift currents could occur.

this domain of particle motion, where their orbits still could be described using guiding center approximation (6). At this schematic picture the non-crossing drifting Larmor circles are the segments of the Speiser orbits before they will reach the $z=0$ plane. The orbits crossing the $z=0$ plane are representing two characteristic populations: meandering portion of Speiser's orbits and the group of trapped particles. The trapped trajectories correspond in our model to the integrable ion orbits (Büchner and Zelenyi, 1989). Near the plane of the field reversal the diamagnetic negative currents coexist with the positive current, which is carried by the meandering part of Speiser's orbits crossing the neutral sheet plane. Diamagnetic current partially compensates the meandering current. The interplay of dia- and meandering effects determines the final profile of the current density inside and outside the CS.

The "meandering" current of "Speiser"particles was estimated in paper by Lyons and Speiser (1985) for the limit of strong anisotropy when $V_{x 0}$ component of the particle velocity is of the order of the plasma flow velocity along the magnetic field lines $V_{D}$. Accordingly their estimate of the total (over the entire thickness of the CS) magnetotail current carried by meandering ions is equal to $I_{y}=\left(4 b n_{0} m_{i} / B_{x 0}\right) V_{x 0}{ }^{2}$, where $I_{y}$ is the total current in the sheet, $n_{0}$ - the density of ions entering the current sheet from one semiplane, $1 \leq b \leq 2$ is the clectron factor, $m_{i}$ is the ion mass, $B_{x 0}$ is the $x$-component of magnetic field outside the sheet, $V_{x 0} \approx V_{D}$ - the plasma drift flow velocity along the magnetic field lines at the CS boundaries in the deHoffmann-Teller frame of refer-

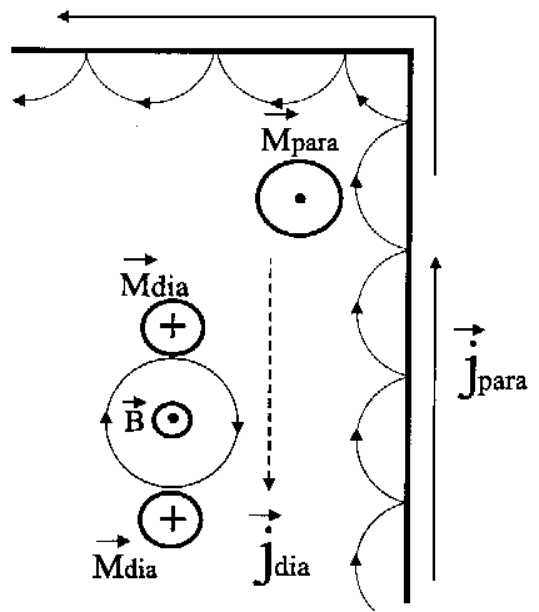

Fig. 2. Schematic picture of the paramagnetic edge current at the boundary of the volume filled by magnetized plasma. $M_{d i a}$ and $M_{\text {para }}$ - magnetic moments of currents carried by particles on Larmor orbits (diamagnetic) and of the current flowing at the boundary of the system. Edge effects could compensate the natural plasma diamagnetism.

ence. For the case of the strong anisotropy of the source distribution their treatment of the corresponding Ohm's law is justified, but for the weak anisotropy contribution of the diamagnetic currents becomes of principle importance and simple Ohm's law like the expression above does not exist.

Meandering particle motion around the $z=0$ plane is principally different from the particle Larmor rotation far from it, corresponding current is flowing in opposite direction to the currents related with the plasma diamagnetism and might be interpreted as paramagnetic. This current is a kind of the "boundary" current flowing along the walls of the bounded plasma system (FrankKamenezkii, 1968). In the $|\Delta z| \sim \rho_{0}$ vicinity of the wall magnetized ions have no room to accomplish the complete circle of Larmor rotation, but instead are reflected (elastically or partially elastically) from the wall surface. As a result the current near the edges is carried only by the segments of Larmor orbits (without the compensation from the particles rotating nearby as it happens far from the "wall"). The plane of magnetic field reversal $(z=0)$, which strongly affects the particle motion, plays the role of such "wall". This analogy of the meandering motion with the edge effects at the plasma boundary is illustrated in Fig. 2 from Frank-Kamenezkii (1968).

Let us estimate approximately the value of the partial contributions of the diamagnetic current outside the sheet into the total current $I_{0}$ in the casc of strong and weak anisotropies, replacing all derivatives by inverse sheet thickness $L^{-1}$. In the limit of strong anisotropy 
we obtain:

$j_{\perp} \approx \frac{c}{B_{0}^{2}}\left(-\frac{p_{\perp} B_{0}}{L}+\frac{p_{\|}}{R^{2}}\left[\vec{R} \times \vec{B}_{0}\right]\right)-c \frac{p_{\perp}}{B_{0} L}$

The values of gradient and magnetization current (first and third terms in Eq.(7)) are small because they are determined by the perpendicular component of velocity, which is small in comparison with $v_{\|} \sim v_{D}$. The parallel velocity gained by the particles due the centrifugal drift is small too. It is of order of $\kappa=B_{n} / B_{o} \sqrt{L / \rho^{*}} \ll 1$ as was shown in paper by Zelenyi and Savenkov [1993]. Therefore the meandering motion is the only essential current carrier in this regime.

In the limit of the weak anisotropy the particle for the first time touches the plane of the magnetic field reversal $z=0$ being still so far from the spatial domain where the field line curvature and magnetic field gradient are strong that one can neglect the values of gradient and centrifugal drifts (first and second terms in Eq.(6)). Therefore the essential carrier of the current near near the edge of the CS will be the magnetization current of non-crossing orbits, described by Eq.(6), and the paramagnetic currents due to "meandering" motion. In this case one can neglect all derivatives $d B / d z$ in Eq.(6), assuming that $B_{0} \approx$ const and leave only $d n / d z$ term in the expression for the magnetization current (last term in Eq.(6)). The competition of paramagnetic and magnetization currents determines the details of the structure of CS profile, particularly the existence of the diamagnetic "wings" and correspondong embedded structure (Fig. 1).

Fig. 3 demonstrates the dimensionless self-consistent magnetic field $b=B(z) / B_{0}$ where $B_{0}$ is the magnetic field outside the sheet for different $\varepsilon=v_{T} / v_{D}$ values. The results of calculations for arbitrary $\varepsilon$ demonstrate that in the cases of moderate and weak anisotropies the self-consistent current sheet equilibrium exists (on the contrary the statement in paper by Holland and Chen (1993)). The sheet thickness is changing with parameter $\varepsilon$ and the profile of magnetic field converges to the limiting profiles for strong and weak anisotropies, assuming the corresponding scaling of Francfort and Pellat (1976) and Ashour-Abdalla et al. (1994).

The comparison of Fig. 4 and Fig. 5 demonstrates that the profiles of current and plasma densities are similar and have the unique scaling in the case of strong anisotropy. In the case of weak anisotropy completely different picture emerges. At large values of $\varepsilon$ the profile of density tends to be almost homogeneous along $Z$-axis. At the same time the corresponding current is concentrated within such plasma sheet (PS) having the characteristic thickness about termal ion gyroradius. We show in Fig. 6 ( $a$ and $b$ ) that the current for $\varepsilon \gg 1$, although having essential paramagnetic kernel $(z \approx 0)$, has also quite long diamagnetic "wings". The absolute value of this "diamagnetic" part of the current is relatively small (ten times less than paramagnetic value $J_{y}(0)$ ) but the

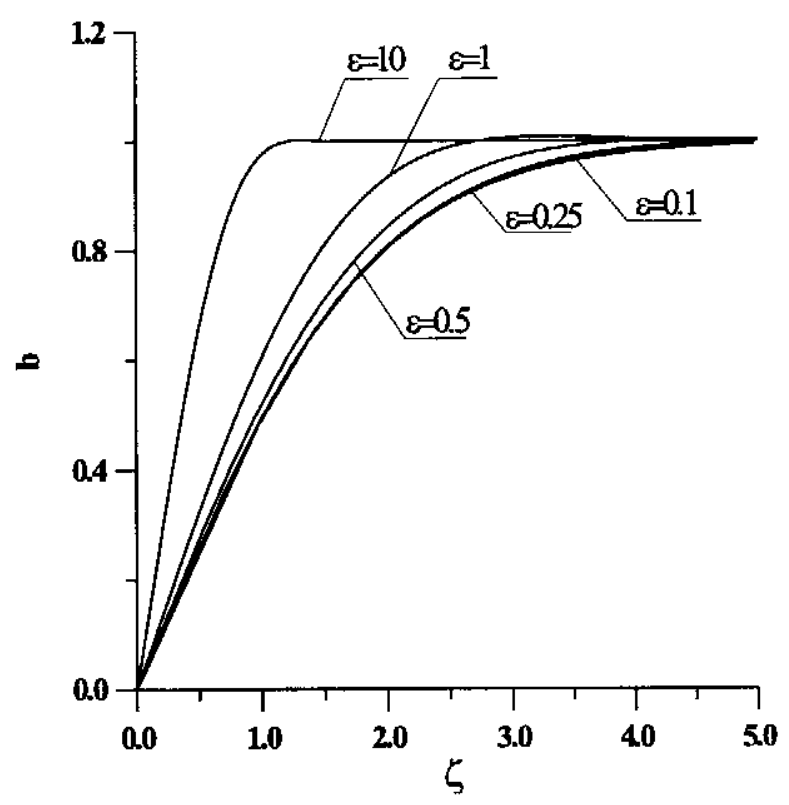

Fig. 3. The self- consistent profiles of the magnetic field $b / b_{0}$ (where $b_{0}$ is a magnetic field outside the sheet) as a function of the dimensionless $Z$-coordinate $\zeta=z \varepsilon^{-4 / 3} \omega_{0} / \nu_{D}$, for various values of the anisotropy parameter $\varepsilon=v_{T} / v_{D}$.

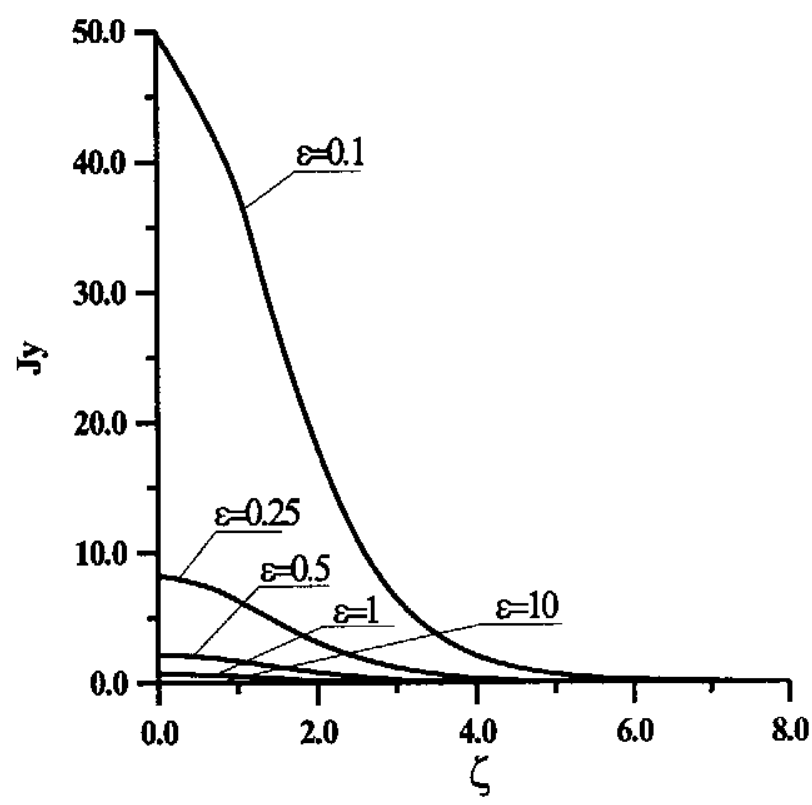

Fig. 4. The dimensionless current density $j_{y}=$ $J_{y}(z) /\left(e n_{0} v_{D} \varepsilon^{2 / 3}\right)$ as a function of the dimensionless $Z$ coordinate $\zeta$ for various values of the anisotropy parameter $\varepsilon=v_{T} / v_{D}$. 


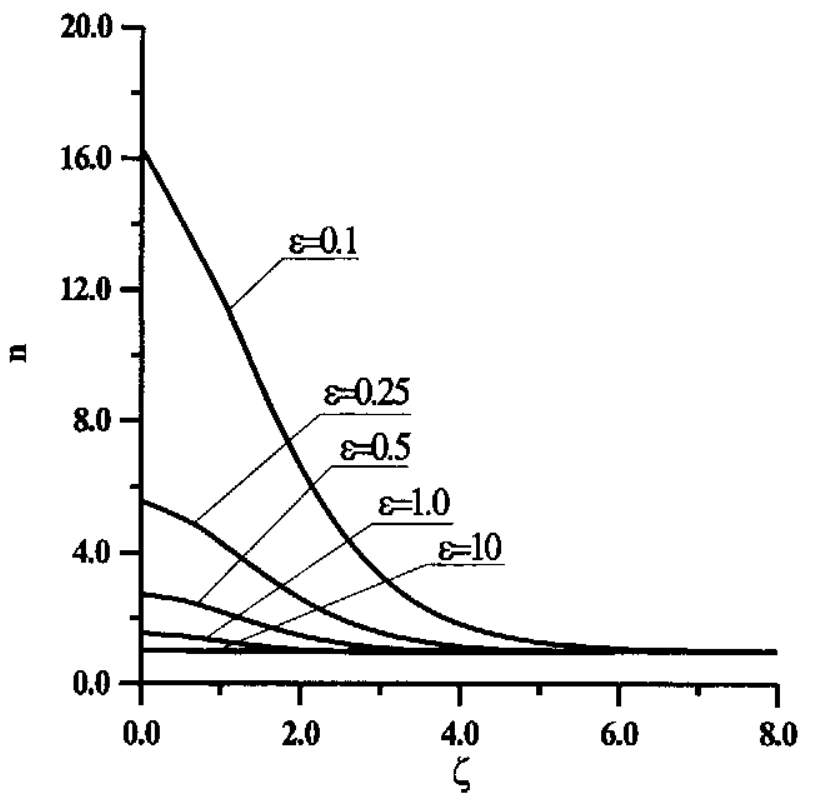

Fig. 5. The dimensionless plasma density $n=n(z) / n_{0}$ (where $n_{0}$ is a plasma density outside the sheet) as a function of the dimensionless $Z$-coordinate $\zeta$ for various values of the anisotropy parameter $\varepsilon=v_{T} / v_{D}$.

existence of these wings results in the difference of profiles of plasma density and cross-tail current in the equilibrium solution.

Fig. 6 a shows that the structure of CS is determined by the process of the compensation of positive and negative components of the current. This result is in good agreement with papers by Holland and Chen (1993), Harold and Chen (1996), where the essential role of diamagnetic currents in the case of weak anisotropy was demonstrated. Fig. 6b illustrates the mechanism of this embedding. Embedding is a characteristic property of the anisotropic CS at $\varepsilon \geq 1$, when the role of diamagnetic currents is dominant everywhere except the near vicinity of the field reversal. In the opposite case of strong anisotropy the influence of diamagnetic currents is decreasing because the motion of particles becomes mostly field-aligned $\left(v_{D} \gg v_{T}\right)$ and diamagnetic effects (proportional to $v_{\perp}^{2}$ ) disappear due to the relative smallness of the Larmor circles. So the current in general has only positive ("paramagnetic") component, and profiles of plasma and current densities approximately coincide.

\section{CS structure for super-strong anisotropy}

We present at Fig. 7 the combined picture of thin ion CSs scaling $L(\varepsilon)$. We distinguish two kinds of thin ion sheets: quasi-adiabatic CSs $\left(\varepsilon>B_{n} / B_{o}\right)$ and nonadiabatic CS $\left(\varepsilon<B_{n} / B_{o}\right)$ which we refer as the superstrong anisotropy case. What is the explanation of the appearance of these two regimes?

In general, quasi-adiabatic $\left(\kappa_{i}<1\right)$ ion, which crosses

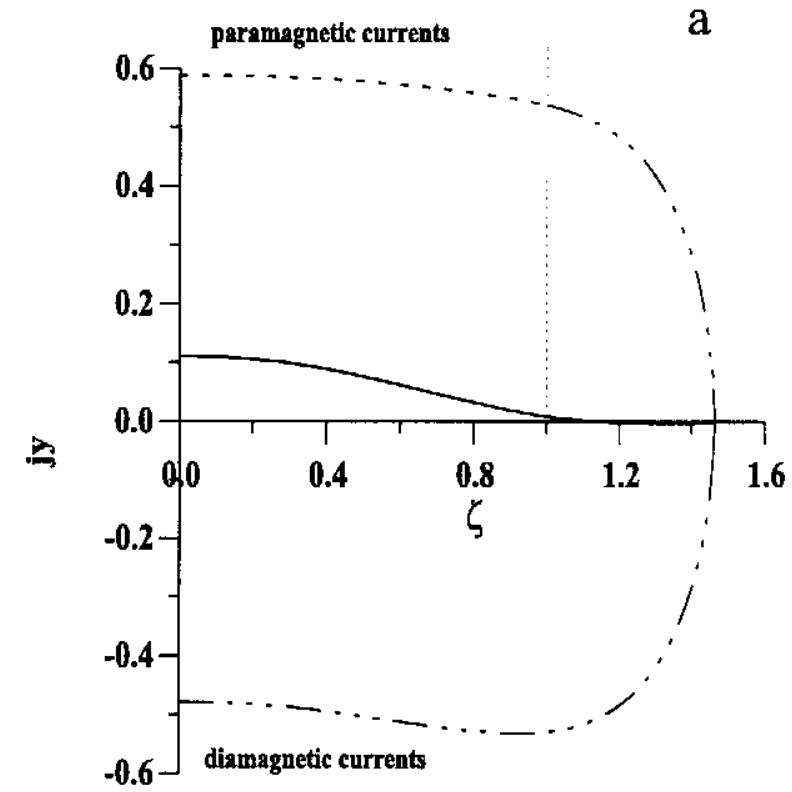

b

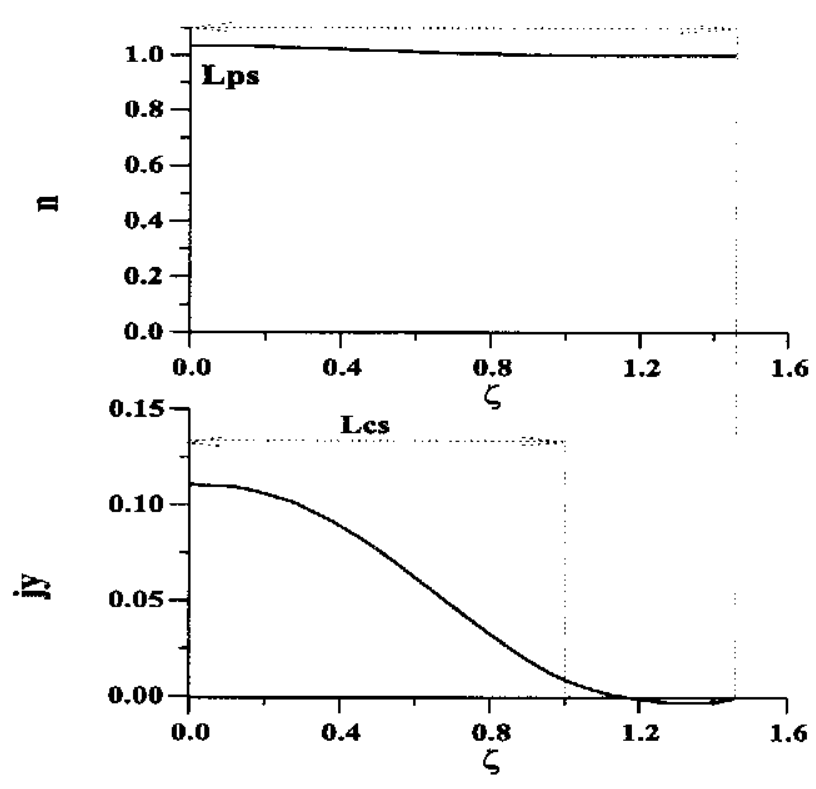

Fig. 6. The scheme of the sheets embedding for weakly anisotropic case: (a) the total current density (solid line) at $\varepsilon=v_{T} / v_{D}=10$ and corresponding partial contributions from dia- (dashed line) and paramagnetic (dotted-dashed line) effects. The vertical dashed line separates regions where only diamagnetic currents exist and the region in the vicinity of the midplane $z=0$ where dia- and paramagnetic currents coexist; (b) the comparison of plasma sheet and current sheet thicknesses $L_{p s}$ and $L_{c s}$ for $\varepsilon=10$ case. 


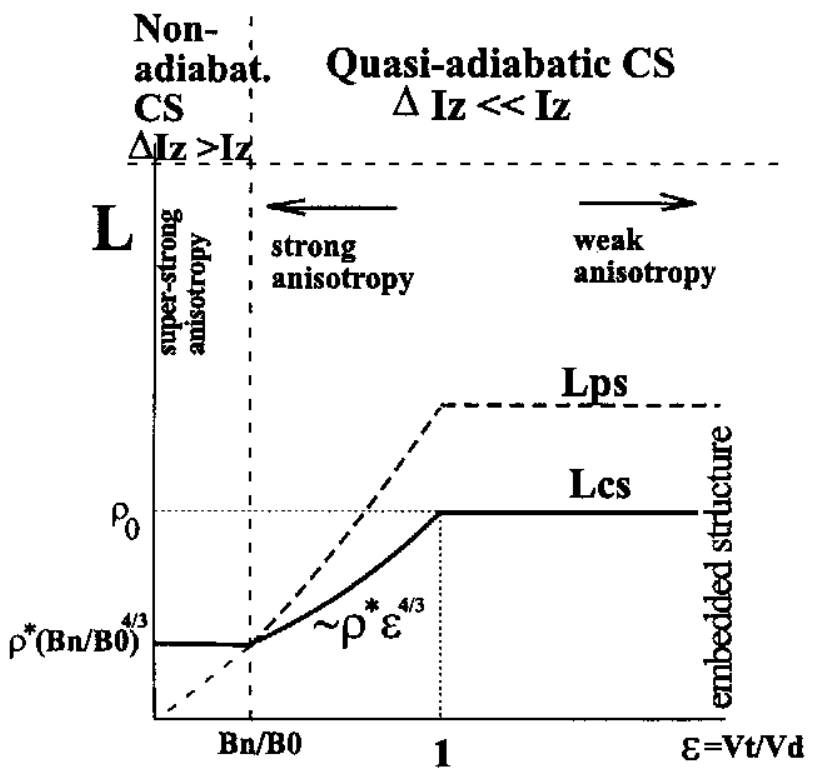

Fig. 7. The combined picture of thin ion current sheets scaling $L(\varepsilon), \varepsilon$ is a source anisotropy, $\rho_{0}=v_{T} / \omega_{0}, \rho^{*}=v_{D} / \omega_{0}, \omega_{0}=$ $e B_{0} / m c$.

the plane of magnetic field reversal, always experiences the jump of quasi-adiabatic invariant $I_{z}$. Magnetic field in the field reversal region has very small radius of curvature and dynamics of field-aligned moving particles $\left(v_{\|}>v_{\perp}\right)$ is controlled by the centrifugal force until their first encounter with CS. It's influence on conservation of CS invariant $I_{z}$ was first estimated by Zelenyi and Savenkov (1993). Then in the series of papers by Delcourt et al., (1994, 1996, 1999) the theory of the particle motion under the influence of sharply peaked centrifugal impulses have been developed for the "chaotic" case at $\kappa=1-3$. As we discussed in the section 1 one could roughly estimate the characteristic average value of the jump according to Büchner and Zelenyi (1989), Zelenyi and Savenkov (1993), Vainshtein et al. (1999) as

$\left|\Delta I^{\prime}\right| \sim \kappa$

where $I^{\prime}$ is the dimensionless value of $I_{z}$ and $\kappa=$ $B_{n} / B_{o} \sqrt{L / \rho^{*}}$ is a parameter of adiabaticity. If the jump of $\left|\Delta I_{z}\right|$ is smaller than the value of $I_{z}$ itself, then our analytical model could more or less adequately describe thin ion CS formation neglecting the non-conservation of $I_{z}$. In the opposite case initial values of $I_{z}$ are small, i.e. particle distribution is almost field-aligned $v_{\perp 0} \ll v_{\| 0} \sim v_{0}$, and our quasi-adiabatic approach could not be applied. To estimate the characteristic value $v_{\perp}^{*}$ which demarcates both limits, let's consider for simplicity the particle starting to move from the boundary of the PS, exactly along field lines of the parabolic magnetic field $B=\left(B_{0}(z / L), 0, B_{n}\right)$. Moving towards the plane $z=0$ particle drifts in the curved magnetic field and gains the additional finite cross-field component of the velocity. This gain of perpendicular velocity due to centrifugal drift, according to paper by Zelenyi and Savenkov (1993), could be easily estimated. At the moment of the first equatorial plane crossing the particle guiding center is reaching the point

$z_{g . c .}^{*}=L b_{n} / \kappa^{2 / 3}$

and $v_{\perp}$ gains the value

$v_{\perp}^{*}=v_{0} \kappa^{2 / 3}$

So particle starting with zero pitch angle $\left(v_{\perp 0}=0\right.$, $I_{z}=0$ ) to the moment of CS crossing acquires the finite value $v_{\perp}^{*}$, which corresponds to $\left|I^{\prime}\right| \sim \kappa$ in agreement with Eq.(8). Therefore the marginal value of the initial velocity $v_{\perp 0}$ should exist, which separates two regimes of anisotropy: a non-adiabatic sheet with super-strong anisotropy where non-conservation of $I_{z}$ is of principal importance and a quasi-adiabatic sheet with the anisotropy although strong, but still satisfying the condition $\left|\Delta I_{z}\right|<I_{z}{ }^{0}$.

To find this we should first estimate the self-consistent CS thickness in the non-adiabatic approximation, i.e. when particles are launched at the boundaries $|z| \sim L$ with very small pitch angles and velocity $v_{\|} \sim v_{D}$. Distance (9) characterizes the width of the meandering region at the moment when particle becomes "crossing" $(z=0$ plane). It is reasonable to estimate the selfconsistent thickness of CS according to this value, i.e. $z_{g . c .}^{*} \cong L$. One could then immediately find the relation between parameter $\kappa$ and normalized $B_{n}$ component of the magnetic field for the self-consistent solution in the form

$b_{n}=\kappa^{2 / 3}$

Using now the definition $\kappa=B_{n} / B_{o} \sqrt{L / \rho^{*}}$ (where $\rho^{*} \simeq$ $\left.v_{D} / \omega_{0}\right)$ one could obtain the value of the CS thickness $L$ as the function of $b_{n}$ and $\rho^{*}$ :

$L=b_{n} \rho^{*}$

So, the marginal value of $v_{\perp 0}$ at the boundary $|z| \sim L$ (for $v_{\perp}<v_{\perp 0}$ one can not neglect the non-conservation of $I_{z}$ ) might be obtained relating the values of $v_{\perp}$ at $z=L$ and $z=z_{\text {g.c. }}{ }^{*}$. Because the motion of particle is still magnetized within this interval $\left(z_{\text {g.c. }}<z<L\right)$ we could obtain:

$\frac{\left(v_{\perp 0}\right)^{2}}{B_{0}} \approx \frac{\left(v_{\perp}^{*}\right)^{2}}{B_{0}\left(z_{g . c .}^{*} / L\right)}$

Taking into account (10)-(12) we could find from (13) that the quasi-adiabatic approximation becomes definitely violated for

$v_{\perp 0} \leq v_{0} b_{n}$

Expression (14) fully conforms with the more rigorous analysis by Zelenyi and Savenkov (1993) and Vainshtein et al. (1999) who developed a general theory 
of the jumps of quasi-adiabatic invariant $I_{z}$ including the limiting case of small initial values of $I^{\prime}<\kappa$, when $\Delta I^{\prime} \rightarrow \kappa>I_{z}$ and jumps of $I_{z}$ already loose their characteristic strong dependence on the initial conditions (resulting in the development of deterministic chaos). Expression (14) is equivalent to $\varepsilon \leq b_{n}$ (as shown in Fig. 6). In this case the sheet structure is determined generally by scattering of ions crossing the plane of magnetic field reversal. Our analytical model developed in previous sections could not be applied in such essentially non-adiabatic limit.

As one can see from Fig. 7, the thickness of ion CS derived from quasi-adiabatic model $\left(L \sim \varepsilon^{4 / 3}\right)$ formally tends to zero in the limit $\varepsilon \rightarrow 0$. In reality this thickness is finite due to unavoidable jumps of the adiabatic invariant. Actually, in the quasi-adiabatic case the thickness is determined by the initial cross-field velocity $v_{\perp}^{*}$ (or equivalently by parameter $\varepsilon$, as was estimated by Francfort and Pellat (1976)). In the non-adiabatic regime, where the initial velocity $v_{\perp 0} \approx 0$, the dynamics of particle meandering motion in the sheet depends upon the abovementioned finite gain of the cross-field velocity $v_{\perp *}$, which determines the effective value of the adiabatic invariant $I_{z}$ at the moment of the first encounter with the field reversal plane $z=0$. Therefore the thickness of the sheet would be also finite and its value is given by Eq.(12). It means that we have found the absolute minimal thickness of the CS created by meandering ion trajectories even if their pitch angles at the boundaries are exactly equal to zero. The ion dominated CS with a smaller thickness could not exist.

This estimate coincides with the estimation of the spatial thickness of the meandering motion in a step-like non self-consistent magnetic field (Alexeev and Malova, 1990) $\mathbf{B}=\left\{B_{x} \operatorname{sign}(z), 0, B_{z}\right\}$. Even if ions initially are moving exactly along the field lines, reaching the equatorial plane, they immediately acquire finite perpendicular velocity because even being field aligned in the upper semiplane $(z>0)$, entering into the lower semiplane $(z<0)$ their motion suddenly gets the finite pitch angle $\alpha \sim 2 B_{n} / B_{0}$ (Fig. 8a). So the width of meandering region in this model is $|\Delta z| \sim \rho_{0} \sim \rho^{*} \alpha$ and even initially step-like $(L \rightarrow 0)$ magnetic field will be smeared to the self- consistent thickness (12) if the system be allowed to evolve to the self-consistent state.

One could also demonstrate (the corresponding details are presented in the Appendix 3 ) that our estimate of the CS thickness (12) does not contradict to the scaling $L^{*}=\rho^{*} b_{n}{ }^{4 / 3}$ discussed in paper by Pritchett and Coroniti (1992). Moreover there is a one-to-one correspondence between these two values because the thickness of super-strong anisotropic CS obtained by Pritchett and Coroniti (1992) and our estimate (12) refer to a different moments of the same meandering motion. The estimate of Pritchett and Coroniti (1992) is the height of the ion trajectory element at the center of the meander semi-circle, while we estimated the maximal CS thick-

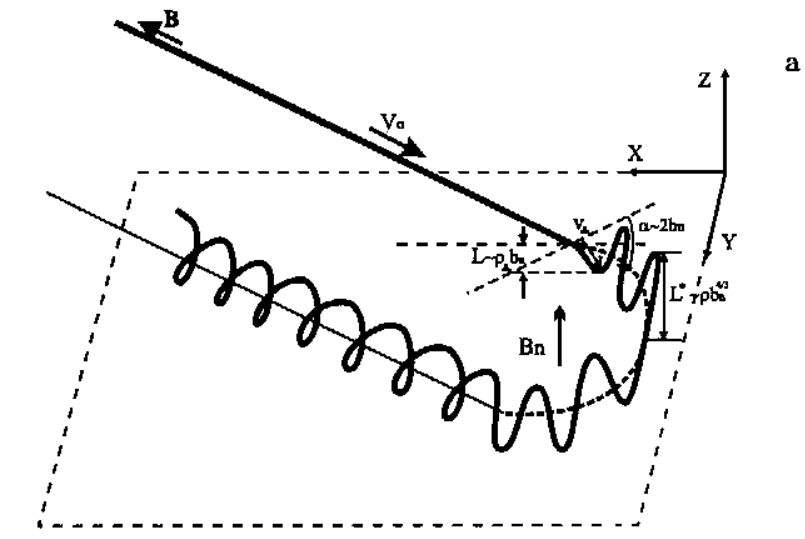

$\mathbf{a}$

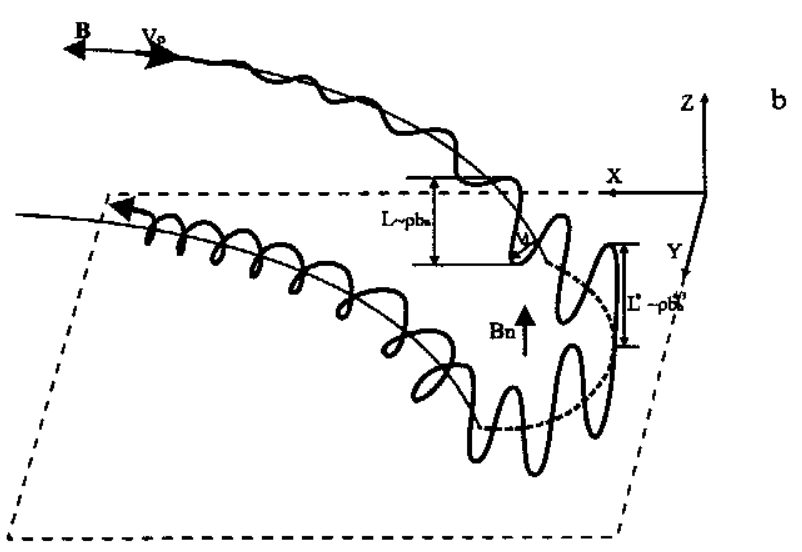

Fig. 8. CS scalings (a) in a step-like non self-consistent magnetic: field $\mathbf{B}=\left\{B_{x} \operatorname{sign}(z), 0, B_{z}\right\}$ [Alexeev et al., 1990, 1995]. Thickness of the meandering region $L=\rho^{*} b_{n}$ is determined by the finite pitch angle $\alpha \sim 2 B_{n} / B_{0}$ that particle acquires at the moment of interaction with CS; (b) self-consistent sheet for the case of super-strong anisotropy of the initial distribution $\left(v_{\perp}<b_{n} v_{\|}\right)$. Sheet is characterized by two thicknesses: $L=\rho^{*} b_{n}$ at the edges of the meandering region near separatrix and $L^{*}=\rho^{*} b_{n}{ }^{4 / 3}$ at the center of the meandering segment of motion. 


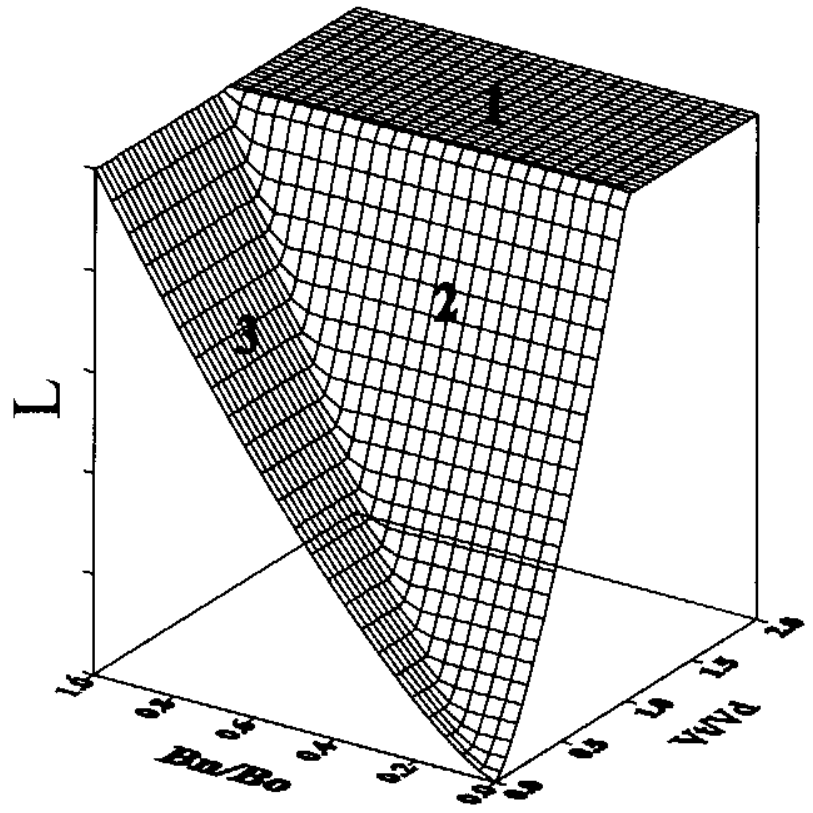

Fig. 9. The scheme of the self- consistent current sheet thickness $L$ as function of parameters $B_{n} / B_{0}$ and $\varepsilon$. The region 1 corresponds to the weak anisotropy scaling $L \sim \rho_{0} \sim v_{T} / \omega_{0}$ (the explanation is given in the text); region 2 corresponds to the strong anisotropy $L \sim \rho_{0} \varepsilon^{l / 3}=\rho^{*} \varepsilon^{4 / 3}$; region 3 reflects the thickness of super-strong anisotropic current sheet $L \sim \rho_{0} \varepsilon^{-1} b_{n}{ }^{4 / 3}=$ $\rho^{*} b_{n}{ }^{4 / 3}$.

ness (12) at the beginning of the meandering regime, when particle just crosses the separatrix. Both estimates relate the thickness of the self-consistent sheet with the scale of the meandering motion. And if our value $L=b_{n} \rho^{*}$ is an upper estimate of the CS thickness, the value $L^{*}=\rho^{*} b_{n}{ }^{4 / 3}$ provides its lowest margin.

As one can see from the comparison of scalings of steplike and "distributed" models in Figs. 8a and 8b our estimate of absolute minimal thickness of CS (Eq.(A17) in the Appendix 3) is in good accordance with the one by Pritchett and Coroniti (1992), which have been obtained from another considerations. Therefore, the absolute minimal thickness of the current sheet is proportional to $b_{n}^{4 / 3}$, and the relation of appropriate scales for the superthin CS is as follows: $L^{*} / L=b_{n}^{1 / 3}$.

\section{The combined picture of thin adiabatic and non-adiabatic current sheets}

The combined scheme of the self- consistent CS thickness $L$ as the function of parameters $b_{n}$ and $\varepsilon$ is presented in Fig. 9. The region 1 corresponds to the approximation of weak anisotropy with the scaling $L \sim$ $\rho_{0} \sim v_{T} / \omega_{0}$. Region 2 corresponds to the scaling obtained earlier by Francfort and Pellat (1976) with $L \approx$ $\rho^{*}\left(v_{T} / v_{D}\right)^{4 / 3}, \rho^{*}=v_{D} / \omega_{0}$. Region 3 reflects the scaling of super-strong anisotropic CS and at the same time (as we argued above) is equivalent to (12). In region 3 CS
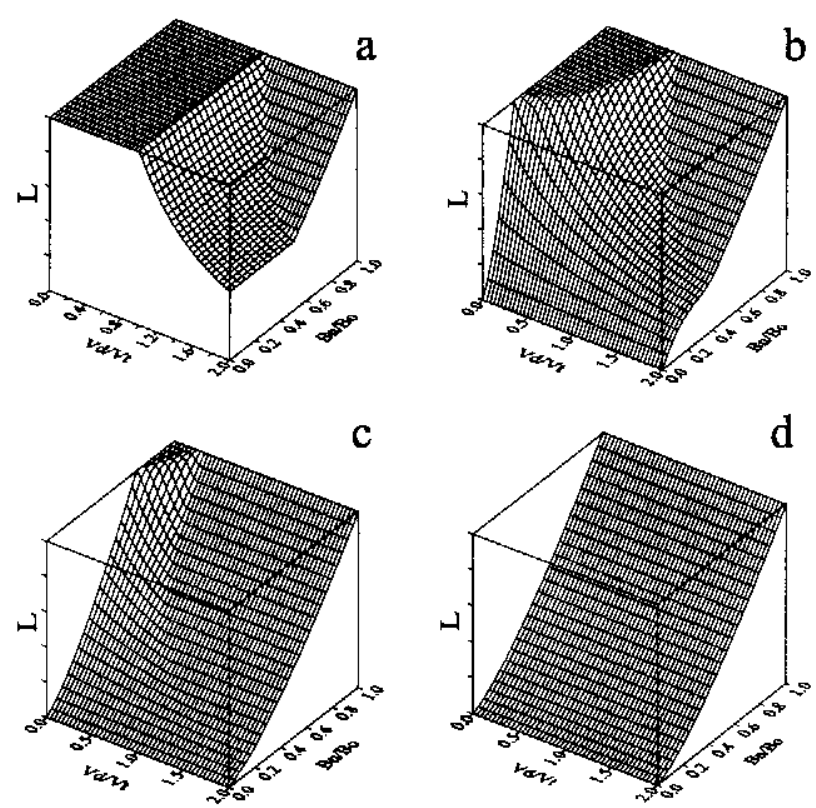

Fig. 10. The dependence of current sheet thickness $L$ from $B_{n} / B_{0}$ and $\varepsilon^{-1}=v_{D} / v_{T}$ parameters for different values of the dimensionless electric field $E y^{*}=c E_{y} /\left(v_{T} B_{0}\right)=0.0(\mathrm{a}), 0.25$ (b), 0.7 (c), 1.0 (d).

thickness doesn't depend on the parameter $\varepsilon$, but is entirely determined by the value of the normal component of magnetic field $B_{n}$. The general thinning of CS due to increase of the plasma anisotropy outside the sheet is in good agreement with the numerical consideration of Harold and Chen, (1996) who investigated the effects of multicomponent distributions on the structure of the CS.

Although our model was considered above in the deHoffman - Teller coordinate frame where the electric field is transformed to zero, we also could easily recalculate our results for the magnetospheric coordinate system where finite dawn-dusk field $E_{y}$ exists. Transferring from deHoffmann - Teller system having velocity $V_{d}=c E_{y} / B_{n}$ we conclude that the anisotropy defined above depends now not only on the initial anisotropy of the distribution function but also on the convection electric field, moreover this effect might be the dominant for the realistic magnetotail parameters.

Let's represent the drift flow velocity introduced by Francfort and Pellat (1976) as a function of the external electric field: $\tilde{V}_{d}=v_{D}+c E_{y} / B_{n}$. Here $v_{D}$ is the velocity of the source ion beam in the magnetospheric frame, $E_{y}$ is dawn-dusk (external) electric field. The dependence of CS thickness $L$ from $b_{n}$ and $\varepsilon^{-1}=v_{D} / v_{T}$ parameters for different values of the dimensionless electric field $E_{y}^{*}=c E_{y} /\left(v_{D} B_{0}\right)$ is shown in Figs. 10a-10d. One can see that the thicknesses characteristic for the regimes of strong and super-strong anisotropy are prevailing for the large values of normalized electric field for almost any values of parameters $b_{n}$ and $\varepsilon=v_{T} / v_{D}$. 
This result is consistent with the generally recognized concept of CS thinning during the beginning of substorm. Turning of $B_{z}$ component of IMF to the South activates the forced reconnection of magnetic field lines at the dayside initiating the magnetic flux pile up at the nightside. During the growth phase the cross-field electric field is growing and plasma convection enhances in the magnetotail. Convective "dawn-dusk" electric field, penetrating into the CS, causes its thinning according to our self-consistent model. At the later stages the development of plasma instabilities and reconnection of magnetic field lines due to current filamentation might, of course, occur. Figs. 10a - 10d support the general view of CS thinning presenting several snapshots of the thin CS scalings for different values of the normalized "dawn-dusk" electric field $E_{y}^{*}$. The essential thinning of the CS in the course of the substorm growth phase could therefore be reasonably well reproduced by our model.

\section{Summary}

Our model discussed above is an analytical development of the kinetic investigations of thin CSs by Speiser (1965), Eastwood (1972), Francfort and Pellat (1976), Burkhart et al. (1992), Pritchett and Coroniti (1992), Holland and Chen (1993), Kropotkin et al. (1997) and other researchers. The evidence of such sheets have been provided recently by in situ measurements in the tail of the Earth's magnetosphere. Two essential types of kinetic models of thin CSs exist now. First, these are full particle-in cell and hybrid simulations by Pritchett and Coroniti (1994, 1995), Hesse and Winske (1996); Hesse et al. (1996) and Kuznetsova et al. (1998) to describe very thin CSs in the magnetotail. Electrons constitute significant or even dominant part of the cross-tail current in such sheets. These models are still restricted because of small ion-to-electron mass ratios and the dependence of results (tearing stability, for example) from the description of electrons as particles or fluid, boundary conditions and other factors. The plasma and current profiles in such models as a rule coincide. Moreover, contrary to the earlier experimental indications of the existence of negatively charged magnetotail CS with electrons as carriers (Mitchell et al., 1990) the later observations by Sergeev et al. (1998) do not confirm these results. Nevertheless such models are very useful in revealing essential features of CS structure and dynamics.

The analytical model presented in this paper is a representative of another class of kinetic models, where the tension of magnetic field lines is balanced by the finite inertia of ions. We have shown that the self-consistent solution of Vlasov- Maxwell equations could be obtained for the anisotropic sheets in the analytical form on the basis of the new set of integrals of ion motion. In a somewhat similar manner the well known isotropic solutions of Harris (1962) and Schindler (1972) have been obtained before. We have generalized the earlier work by Kropotkin et al. (1997), so that our basic equation of Grad-Shafranov type describes the cases of both weak and strong (but not super-strong) anisotropy of the initial distribution function. The characteristic property of self-consistent CS structure predicted by this model is the embedding of CS inside the PS.

We demonstrated that the maximum thickness of the CS is achieved in the case of weak anisotropy and is of the order of the thermal gyroradius of ions outside the sheet. With the increase of anisotropy the sheet becomes compressed down to some finite limiting thickness. We have estimated that this smallest possible thickness of the sheet in the limit of the super-strong anisotropy $v_{T} / v_{D}<b_{n}$ is in the range $\rho^{*} b_{n}^{4 / 3}<\dot{L}<$ $\rho^{*} b_{n}$. In this regime the CS structure is determined by nonadiabatic effects ( $I_{z}$ is essentially non conserved) and consists mainly of paramagnetic currents. Existence of the external electric field $E_{y}$ results in the effective increase of anisotropy and we considered the influence of $E_{y}$ on the CS scaling. The current sheet in our model thins with the growth of the dawn-dusk electric field and this effect might have important implications for the dynamics of substorms.

\section{Appendix 1. The basic equations}

To relate the distribution (5) with its asymptotic form outside the sheet we use the relation $I=(2 m c / e) \mu$ between the sheet invariant $I$ and the magnetic moment $\mu=m v_{\perp}^{2} /(2 B)$ at the edge of the sheet. Using the Liuville theorem we could represent the ion distribution in the form

$f=\frac{n_{0} \exp \left\{-\varepsilon^{-2 / 3}\left[\left(\sqrt{w_{0}^{2}-I}-\varepsilon^{-2 / 3}\right)^{2}+I\right]\right\}}{\pi^{3 / 2} v_{T}^{3}\left[1+\operatorname{erf}\left(\varepsilon^{-1}\right)\right]}$

where $\varepsilon=v_{T} / v_{D}, \mathbf{w} \equiv \varepsilon^{-2 / 3} \mathbf{v} / v_{D}, w_{0}=|\mathbf{w}|, I=$ $\varepsilon^{2 / 3} I_{z} \omega_{0} /\left(m v_{T}\right), n_{0}$ is the plasma density outside the sheet which is created by two counterstreaming beams, $\omega_{0}=e B_{0} /(m c)$.

Using (A1) and introducing the dimensionless variables $\zeta=z \epsilon^{-4 / 3} \omega_{0} / v_{D}, \zeta_{0,1}=z_{0,1} \epsilon^{-4 / 3} \omega_{0} / v_{D}$, and $b=B / B_{0}$, as well as their transforms $b(\zeta) \rightarrow b(a)$ $\rightarrow \gamma(\eta)$ with

$$
\begin{aligned}
a & =\int_{0}^{\zeta} b\left(\zeta^{\prime}\right) d \zeta^{\prime}, \eta=\varepsilon^{2 / 3} a, \gamma(\eta)=b(a), \\
\frac{d b}{d \zeta} & =\frac{d b}{d a} \frac{d a}{d \zeta}=\frac{1}{2} \frac{d b^{2}}{d a}
\end{aligned}
$$

we arrive to the following dimensionless equation for the sheet magnetic field $\gamma$ as a function of the nontrivial part $\eta \sim \int B\left(z^{\prime}\right) d z$ of the vector-potential in the region $\eta \geq 0$

$\gamma^{2}(\eta)=\frac{8 \varepsilon^{1 / 3}}{\pi^{3 / 2}}\left(\frac{v_{D}}{v_{A}}\right)^{2} \frac{F_{(+)}(\eta)+F_{(-)}(\eta)}{1+\operatorname{erf}\left(\varepsilon^{-1}\right)}$ 
where

$$
\begin{aligned}
& F_{( \pm)}(\eta)= \pm \int_{0}^{\eta} d \eta^{\prime} \int_{0}^{\infty} d w_{x} \int_{0}^{\infty} w_{y} d w_{y} \int_{0}^{\infty} d w_{\zeta} \\
& \exp \left\{-\varepsilon^{-2 / 3}\left[\left(\sqrt{w_{x}^{2}+w_{y}^{2}+w_{\zeta}^{2}-I_{ \pm}}-\varepsilon^{-2 / 3}\right)^{2}+I_{ \pm}\right]\right\}
\end{aligned}
$$

where $w_{\zeta}=d \zeta / d t$ and the difference between the contributions with positive and negative dawn-dusk components of the particle velocity is only in the sign of the whole expression and the form of the invariants $I_{ \pm}$

$I_{ \pm}=\frac{2}{\pi} \int_{\eta_{0 \pm}}^{\eta_{ \pm}} \sqrt{w_{y}^{2}+w_{\zeta}^{2}-\left( \pm w_{y}+\eta^{\prime \prime \prime}-\eta^{\prime}\right)^{2}} \frac{d \eta^{\prime \prime \prime}}{\gamma\left(\eta^{\prime \prime \prime}\right)}$

where

$\eta_{0 \pm}=\max \left\{0, \eta^{\prime}-\sqrt{w_{y}^{2}+w_{\varsigma}^{2}} \mp w_{y}\right\}$

and

$\eta_{ \pm}=\sqrt{w_{y}^{2}+w_{\zeta}^{2}}+\eta^{\prime} \mp w_{y}$

The parameter $v_{A} / v_{D}$ must satisfy the boundary relation $\gamma(\eta \rightarrow \infty)=1$

$$
\begin{aligned}
\frac{v_{A}}{v_{D}}= & \frac{2 \sqrt{2}}{\pi^{3 / 4}} \frac{\varepsilon^{1 / 6}}{\sqrt{\left(1+e r f\left(\varepsilon^{-1}\right)\right)}} \\
& \sqrt{\int_{0}^{\infty} d \eta^{\prime}\left(F_{(+)}\left(\eta^{\prime}, w_{x}\right)+F_{(-)}\left(\eta^{\prime}, w_{x}\right)\right)}
\end{aligned}
$$

Equation (A8) corresponds to the pressure balance condition in a sheet and should be satisfied for the selfconsistent solution (Burkhart et al., 1992).

\section{Appendix 2. Limiting cases of the basic theory}

In the limiting case $\varepsilon \rightarrow 0$ we could estimate $w_{0} \sim$ $\varepsilon^{-2 / 3} \rightarrow \infty, I_{+} \sim \varepsilon^{2 / 3} \rightarrow 0, I_{-} \sim w_{y}^{2} \rightarrow \infty$ and after the restoration of variables $a$ and $b$ given by (A2) the basic equation (A3) may be reduced to the following parameterless form

$$
\begin{aligned}
b^{2}(a)= & \frac{2 \sqrt{2}}{\pi} \int_{0}^{a} d a^{\prime} \int_{0}^{\pi / 2}(\cos \phi)^{3 / 2} d \phi \\
& \int_{a^{\prime}}^{\infty} \frac{d a^{\prime \prime}}{\sqrt{a^{\prime \prime}-a^{\prime}}} \exp \left(-I_{+}^{(s)}\right)\left(\phi, a^{\prime \prime}\right)
\end{aligned}
$$

with

$I_{+}^{(s)}=\frac{2^{3 / 2}(\cos \phi)^{1 / 2}}{\pi} \int_{0}^{a^{\prime \prime}} \sqrt{a^{\prime \prime}-a^{\prime \prime \prime}} \frac{d a^{\prime \prime \prime}}{b\left(a^{\prime \prime \prime}\right)}$

which is in fact represents the universal equation for strongly anisotropic sheet obtained earlier by Kropotkin et al.(1997). According to Eqs.(A2) and (A3) the corresponding current and magnetic field profiles have the characteristic scaling $L \sim \varepsilon^{4 / 3} v_{D} / \omega_{0}$, which was predicted earlier by Francfort and Pellat (1976) on the basis of non self-consistent approach.

In the other limit $\varepsilon \rightarrow \infty$ the parameters $\eta, w_{x}, w_{y}$, $w_{\zeta}$, and $I_{ \pm}^{1 / 2}$ scale as $\varepsilon^{1 / 3}$. As a result expanding exponent in the right hand side of (A4) and using the new set of variables $\gamma(\eta)=\beta(\alpha), \alpha=\varepsilon^{-1 / 3} \eta=\varepsilon^{1 / 3} a$, one can obtain the following equation like (A9)-(A10)

$$
\begin{aligned}
\beta^{2}(\alpha)= & (16 / \pi) \int_{0}^{\infty} r^{2} \exp \left(-r^{2}\right) d r \int_{0}^{\pi / 2} \sin \theta d \theta \int_{0}^{\pi / 2} d \varphi \\
& \left(\int_{0}^{2 y}-\int_{\alpha}^{\alpha+2 y}\right) d \alpha^{\prime} \sqrt{r^{2}-I_{+}^{(w)}\left(\alpha^{\prime}, y, z\right)} \quad(\mathrm{A} 11)
\end{aligned}
$$

where

$$
\begin{aligned}
I_{+}^{(w)}\left(\alpha^{\prime}, y, z\right)= & \frac{2}{\pi} \int_{\max \left\{0, \alpha^{\prime}-\sqrt{y^{2}+z^{2}}-y\right\}}^{\sqrt{y^{2}+z^{2}}+\alpha^{\prime}-y} \\
& \sqrt{y^{2}+z^{2}-\left(y-\alpha^{\prime}+\alpha^{\prime \prime}\right)^{2}} \frac{d \alpha^{\prime \prime}}{\beta\left(\alpha^{\prime \prime}\right)}
\end{aligned}
$$

and $x=r \sin \theta \cos \varphi, y=r \sin \theta \sin \varphi, z=r \cos \theta$. In particular, according to (A11), the coordinate $\alpha$ is of the order of $\alpha \sim \varepsilon^{1 / 3} \zeta \sim 1$. Therefore, contrary to the case of strong anisotropy the corresponding profiles of current density and magnetic field scale as $L \sim \varepsilon v_{D} / \omega_{0}=\rho$.

\section{Appendix 3. Estimates of the scalings of the CS thickness for super-strong anisotropy}

The correspondence between two scaling parameters (expression (12) and the scaling $L \sim b_{n}^{4 / 3}$ ) can be easily found from simple calculations. Let's consider the ion moving in the curved magnetic field towards the sheet plane with zero pitch angle at the boundary. As we argued above at the moment when the particle enters the sheet its perpendicular velocity due to gradient drift is finite and equal to (10). Taking into account the upper estimate (12), one can suppose that entering into the CS the particle is moving in the field where the size of the field inhomogeneity (which is of the order of the sheet thickness) is smaller than Larmor radius $\rho$, i.e. $|\operatorname{grad} \mathbf{B} / \mathbf{B}|^{-1} \ll \rho$, so on a scale $\sim \rho$ we might approximately consider the profile of the magnetic field as a step-like function. One might relate then the perpendicular pitch-angle of particle $\theta_{0}$ near the separatrix and the phase $\psi$ of Larmor rotation at the moment when the particle crosses the equatorial plane $z=0$, being at the center of its meandering semicircle $\left(\left\langle v_{x}\right\rangle=0\right)$. Following the reasoning of estimates by Francfort and Pellat (1976)

$(\pi-\psi)^{3} \approx \sin ^{2} \theta_{0}$

where

$\sin \theta_{0} \approx v_{\perp} / v_{0}$ 
and $v_{\perp}$ is the value of perpendicular velocity just before the separatrix crossing. Due to simple geometric consideration the height $L^{*}$ of the quasi-adiabatic element of the orbit at the center of the meandering circle in the plane $z=0$ is of the order of

$L^{*} \approx \rho^{*}(\pi-\psi)^{2}$

Substituting (A13) and (A14) to (A15) one might get

$L^{*} \approx \rho^{*} \sin ^{4 / 3} \theta_{0} \sim \rho^{*}\left(v_{\perp} / v_{0}\right)^{4 / 3}$

Taking into account the estimate of the perpendicular component of velocity (10) at the moment of separatrix crossing and expression (11) we obtain from (A16) that

$L^{*} \approx \rho^{*} b_{n}{ }^{4 / 3}$

This value coincides with estimate by Pritchett and Coroniti (1992) of the CS scaling in the case of super-strong anisotropy.

Acknowledgements. The authors are grateful to Drs. D. Delcourt and V. Sergeev for helpful discussions.

This work was supported by the Russian Basic Research Foundation grants 97-02-16489, 99-05-65567, INTAS grants 97-1612, 00-0078 and the grant of Basic Research Competitive Center of Ministry of Education of Russian Federation.

\section{References}

Alexeev, I.I., and H.V. Malova, The plasma sheet structure in the magnetotail (in Russian), Geomagn. Aeron., 30, 407, 1990.

Alexeev, I.I., and H.V. Malova, On the model of current sheet in the magnetosphere tail, taking into account the interaction of transit and traped particles, Advances in Space Research, 16, $205,1995$.

Ashour-Abdalla, M., L.M. Zelenyi, V. Peroomian, and R.L. Richard, Consequences of magnetotail ion dynamics, J. Geophys. Res., 99, 14,891, 1994.

Ashour-Abdalla, M., L.A. Frank, W.R. Paterson, V. Peroomian, and L. M. Zelenyi, Proton velocity distributions in the magnetotail: theory and observations, J. Geophys. Res., 101, 2587, 1996.

Birn, J., Magnetotail equilibrium theory: The general threedimensional solution, J. Geophys. Res., 92, 11,101, 1987.

Büchner, J., and L.M.Zelenyi, Regular and chaotic charged particle motion in magnetotaillike field reversals 1. Basic theory of trapped motion, J. Geophys. Res., 94, 11821, 1989.

Burkhart, G.R., J.F. Drake, P.B. Dusenbery, and T.W. Speiser, A particle model for magnetotail neutral sheet equilibria, $J$. Geophys. Res., 97, 13,799, 1992.

Burkhart, G.R., and J. Chen, Differential memory in the Earth's magnetotail, J. Geophys. Res., 97, 6479, 1992.

Delcourt, D.C., and R.F. Martin Jr., Application of the centrifugal impulse model to particle motion in the near-Earth plasma sheet, J. Geophys. Res., 99, 23,583, 1994.

Delcourt, D.C., G. Belmont, J.-A. Sauvaud, T.E. Moore and R. F. Martin Jr., Centrifugally driven phase bunching and related current sheet structure in the near-Earth magnetotail, J. Geophys. Res., 101, 19,839, 1996.

Delcourt, D.C., and R.F. Martin Jr., Pitch angle scattering near energy resonances in the geomagnetic tail, J. Geophys. Res., $104,383,1999$.

Eastwood, J.W., Consistency of fields and particle motion in the "Speiser" model of the current, sheet, Planet Space Sci., 20, $1555,1972$.
Fairfield, D.H., Magnetotail energy storage and the variability of the magnetotail current sheet, in Magnetic Reconnection in Space and Laboratory Plasmas, Geophys. Monogr. Ser., vol. 30, edited by E. W. Hones, p. 168, AGU, Washington, D. C., 1984.

Francfort, P., and R. Pellat, Magnetic merging in collisionless plasmas, Geophys. Res. Lett., 3, 433, 1976.

Frank-Kamenezkii D.A., in The lectures on plasma physics (in Russian), Moscow, Atomisdat, 1968.

Harold, J.B., and J. Chen, Kinetic thinning in one- dimensional self-consistent current sheets, J. Geophys. Res., 101, 24,899, 1996.

Harris, E.G., On a plasma sheath separating regions of oppositely directed magnetic fields, Nuovo Chimento, 23, 115, 1962.

Hesse, M., and D. Winske, Hybrid modeling of the formation and structure of thin current sheets in the magnetotail, in Proceedings of the Third International Conference on Substorms (ICS-3), Spec. Publ. ESA SP-389 p. 231, Eur. Space Agency, Paris, 1996.

Hesse, M., D. Winske, M.M. Kuznetsova, J. Birn, and K. Schindler, Hybrid modeling of the formation of thin current sheets in magnetotail configurations, J. Geomagn. Geoelectr., $48,749,1996$.

Holland, D.L., and J. Chen, Self-consistent current sheet structures in the quiet-time magnetotail, Geophys. Res. Lett., 20, $1775,1993$.

Kan, J.R., On the structure of the magnetotail current sheet, $J$. Geophys. Res., 78, 3773, 1973.

Kaufmann, R. L., Substorm currents: Growth phase and onset, J. Geophys. Res., 92, 7471, 1987.

Kaufmann, R.L., I.D. Kontodinas, B.M. Ball, and D. J. Larson, Nonguiding center motion and substorm effects in the magnetotail, J. Geophys. Res., 102, 22,155, 1997.

Kropotkin, A.P., H.V. Malova, and M.I. Sitnov, Self-consistent structure of a thin anisotropic current sheet, J. Geophys. Res., $102,22,099,1997$.

Kuznetsova, M.M., M. Hesse, and D. Winske, Kinetic quasiviscous and bulk flow inertia effects in collisionless magnetotail reconnection, J. Geophys. Res., 103, 199, 1998.

Larsson, D.J., R.L. Kaufmann, Structure of magnetotail current sheet, J. Geophys. Res., 101, 21, 447, 1996.

Lembege, B. and R. Pellat, Stability of a thick two-dimensional quasineutral sheet, Phys. Fluids, 25, 1995, 1982.

Lyons, L.R. and T.W. Speiser, Ohm's law for a current sheet, $J$. Geophys. Res., 90, 8543, 1985.

McPherron, R.L., A. Nishida, and C.T. Russell, Is near-Earth current sheet thinning the cause of auroral substorm onset? in Quantitative Modeling of Magnetosphere-Ionosphere Coupling Processes, edited by Y. Kamide and R. A. Wolf, pp.252-265, Kyoto Sangyo University, Kyoto, Japan, 1987.

Mitchell, D.G., G.J. Williarns, C.Y. Huang, L.A. Frank, and C. T. Russell, Current carriers in the near-Earth cross-tail current sheet during substorm growth phase, Geophys. Res. Lett., 17, $583,1990$.

Nishikawa, K.I, Reconnection at near Earth magnetotail and substorm studied by 3-D EM particle code. In: "Geospace mass and energy flow results from ISTP Program", ed. J. L. Horwitz ,W. K. Peterson, and D. J. Gallagher, AGU Geophys Monograph., 104, p.175, 1998.

Pritchett, P.L., and F.V. Coroniti, Formation and stability of the self-consistent one-dimensional tail current sheet, $J$. Geophys. Res., 97, 16,773, 1992.

Pritchett, P.L., and F.V. Coroniti, Convection and the formation of thin current sheets in the near-Earth plasma sheet, Geophys. Res. Lett., 21, 1587, 1994.

Pritchett, P.L., and F.V. Coroniti, Formation of thin current sheets during plasma sheet convection, J. Geophys. Res., 100, $23,551,1995$. 
Pulkkinen, T.I., D.N. Baker, C.J. Owen, J.T. Gosling, and N. Murthy, Thin current sheets in the deep geomagnetotail, Geophys. Res. Lett., 20, 2427, 1993.

Pukkinen, T.I., D.N. Baker, L.L. Cogger, 'T. Mukai, and H.J. Singer, Coupling of inner and midtail processes, in: SUBSTORMS-4, Ed. by S. Kokubun and Y. Kamide, Terra Scientific Publishing Company/Kluwer Academic Publishers, p.749, 1998.

Sanny, J., R.L. McPherron, C.T. Russell, D.N. Baker, T.I. Pulkkinen, and A. Nishida, Growth phase thinning of the near-Earth current sheet during the CDAW-6 substorm, J. Geophys. Res., $99,5805,1994$.

Savenkov, B.V., L.M. Zelenyi, M. Ashour-Abdalla, and J. Büchner, Regular and chaotic aspects of charged particle motion in a magnetotail-like field with a neutral line, Geophys. Res. Lett., $18,1587,1991$.

Schindler, K., A self-consistent theory of the tail of the magnetosphere, in Earth's Magnetospheric Processes, edited by B. M. McCormac, p.200, D. Reidel, Norwell, Mass., 1972.

Schindler, K., A theory of the substorm mechanism, J. Geophys. Res., 79, 2803, 1974.

Sergeev, V.A., D.G. Mitchell, C.T. Russell, and D.J. Williams, Structure of the tail plasma/current sheet at $11 \mathrm{Re}$ and its changes in the course of a substorm, J. Geophys. Res., 98, $17345,1993$.

Sergeev, V.A., T.I. Pulkkinen, and R.J. Pellinen, Coupled mode scenario for the magnetospheric dynamics, J. Geophys. Res., 101, 13,047, 1996.

Sergeev, V.A., V. Angelopoulos, C. Carlson, and P. Sutcliffe, Current sheet measurements within a flapping plasma sheet, $J$. Geophys. Res., 103, 9177, 1998.
Shay, M.A., J.F. Drake, R.E. Denton., D. Biscamp, Sructure of dissipation region during collisionless magnetic reconnection $J$. Geophys. Res., 103, 9165, 1998.

Sitnov, M.I., H.V. Malova, and A.S. Sharma, Linear stability of a tearing mode in a quasi-neutral current sheet, Plasma. Phys. Rep. (transl. from Fizika Plazmy), 25, 227, 1999.

Sitnov, M.I., L.M. Zelenyi, H.V. Malova, and A.S. Sharma, Thin current sheet embedded within a thicker plasma sheet: Selfconsistent kinetic theory, J. Geophys. Res. (in press), 2000.

Smets, R., and D. Delcourt, Ion and electron distribution functions in the distant magnetotail: modeling of Geotail observations, J. Geophys. Res., 103, 20,407, 1998.

Sonnerup, B.U.O., Adiabatic particle orbits in a magnetic null sheet, J. Geophys. Res., 76, 8211, 1971.

Speiser, T.W., Particle trajectories in model current sheets; 1. Analytical solutions, J. Geophys. Res., 70, 4219, 1965.

Speiser, T.W., Conductivity without collisions or noise, Planet. Space Sci., 18, 613, 1970.

Zelenyi, L.M., and B.V. Savenkov, Violating of quasiadiabaticity during the particle motion in magnetic field configurations with strongly curved field lines, Plasma. Phys. Rep. (Transl.from Russian), 19, 712, 1993.

Zelenyi, L.M., A.V.Milovanov, and G. Zimbardo, Multiscale magnetic structure of the distant tail: self-consistent fractal approach, New perspectives on the Earth's magnetotail, Geopys Monogr.Ser., vol. 105, edited by A. Nishida, D.N.Baker, and S.W.H.Cowley, p. 321, AGU, Washington, D. C., 1998.

Vainshtein, D.L., L.M. Zelenyi, A.I. Neishtadt, and B.V. Savenkov, The jumps of adiabatic invariant at its small initial values, Plasma. Phys. Rep. (in press), 1999. 